\title{
THE ULTRAVIOLET SPECTRA OF \\ OLD STELLAR POPULATIONS
}

\author{
BEN DORMAN \\ NASA/Goddard Space Flight Center \\ AND \\ ROBERT W. O'CONNELL \\ Astronomy Department, University of Virginia
}

We investigate the utility of UV spectra of old stellar populations as diagnostics of galaxy properties. For $\lambda<3800 \AA$, the integrated light of old stellar populations is dominated by two components: the hot stars which give rise to the UV upturn phenomenon at far-UV wavelengths, and the stars (i.e. main sequence, and subgiants) that lie closest to the turnoff. The mid-UV radiation from the turnoff varies strongly with metallicity, and less so with age; mid-UV light must be correct for the effect of the independent far-UV upturn component, but this is straightforward. Population synthesis models that account for the flux from the UV upturn can therefore determine the characteristics of the underlying stellar content from the mid-UV spectral region. The age \& metallicity dependence of the far-UV $1550 \AA$ is not well understood (see Dorman, O'Connell \& Rood 1995, ApJ 442,105) since the mass loss mechanism on the RGB that produces very blue HB stars has no physical model. In contrast mid-UV indicators derive from the turnoff population which best represents the quantities we wish to measure, and are in addition visible at favourable optical wavelengths for $z \lesssim 1$.

We are investigating various spectral diagnostics with simple (single burst, single metallicity, Salpeter IMF) population models that include varying strengths of UV upturn. We find that a broadband index centered around $2200 \AA$ measures a spectral region in which there are no strong lines and the continuum is very metal-sensitive. Models of metallicities $[\mathrm{Fe} / \mathrm{H}]=-0.47,0.0, \& 0.3$ occupy almost disjoint locations on a $15-V$ vs $22-V$ diagram, potentially helping to resolve the age/metallicity degeneracy. Higher precision data than is possible with the IUE satellite may be able to place (differential) ages on luminosity weighted galaxy populations. 